\title{
GRB 990704: The most X-ray rich BeppoSAX gamma-ray burst
}

\author{
M. Feroci ${ }^{1}$, L. A. Antonelli ${ }^{2}$, P. Soffitta ${ }^{1}$, J. J. M. in 't Zand ${ }^{3}$, L. Amati ${ }^{4}$, E. Costa ${ }^{1}$, L. Piro $^{1}$, \\ F. Frontera ${ }^{4,5}$, E. Pian ${ }^{4,6}$, J. Heise $^{3}$, and L. Nicastro ${ }^{7}$ \\ 1 Istituto Astrofisica Spaziale, C.N.R., Area di Tor Vergata, Via Fosso del Cavaliere 100, 00133 Roma, Italy \\ 2 Osservatorio Astronomico di Roma, Via Frascati 33, 00040 Monteporzio Catone, Italy \\ 3 Space Research Organization in the Netherlands, Sorbonnelaan 2, 3584, CA Utrecht, The Netherlands \\ 4 Istituto Tecnologie e Studio Radiazioni Extraterrestri, CNR, Via Gobetti 101, 40129 Bologna, Italy \\ 5 Dipartimento di Fisica, Università di Ferrara, Via Paradiso 12, 44100 Ferrara, Italy \\ 6 Osservatorio Astronomico di Trieste, Via G.B. Tiepolo 11, 34131, Trieste, Italy \\ 7 Istituto di Fisica Cosmica ed Applicazioni dell'Informatica, CNR, Via Ugo La Malfa 153, 90138 Palermo, Italy
}

Received 30 March 2001 / Accepted 22 August 2001

\begin{abstract}
We present the X- and $\gamma$-ray detection of GRB 990704 and the discovery and study of its X-ray afterglow, 1SAX J1219.5-0350. Two pointed BeppoSAX observations with the narrow field instruments were performed on this source, separated in time by one week. The decay of the X-ray flux within the first observation appears unusually slow, being best-fit by a power law with negative index $0.83 \pm 0.16$. Such a slow decay is consistent with the non-detection in our second observation, but its back-extrapolation to the time of the GRB largely underestimates the detected GRB X-ray prompt emission. In addition, the GRB prompt event shows, among the BeppoSAX-WFC detected sample, unprecedentedly high ratios of $\mathrm{X}$ - and gamma-ray peak fluxes $\left(F_{2-10 \mathrm{keV}} / F_{40-700 \mathrm{keV}} \sim 0.6\right.$, and $\left.F_{2-26 \mathrm{keV}} / F_{40-700 \mathrm{keV}} \sim 1.6\right)$ and fluences $\left(S_{2-10 \mathrm{keV}} / S_{40-700 \mathrm{keV}} \sim 1.5\right.$ and $\left.S_{2-26 \mathrm{keV}} / S_{40-700 \mathrm{keV}} \sim 2.8\right)$, making it, among the BeppoSAX arcminute-localized GRBs, the closest to the recently discovered class of Fast X-ray Transients.
\end{abstract}

Key words. gamma-rays: bursts - stars: individual: 1SAX J1219.5-0350

\section{Introduction}

Despite the huge step forward allowed by the BeppoSAX discovery of the X-ray afterglows (Costa et al. 1997) of gamma-ray bursts (GRBs) and by the related discoveries of optical (van Paradijs et al. 1997) and radio afterglows (Frail et al. 1997), the ultimate explanation of GRBs remains unknown. Several sources associated with GRBs have been demonstrated to be at cosmological distances (e.g., see Costa 2000; Lamb 2000 for reviews), but the current sample only includes long duration GRBs leaving open the possibility of the existence of different populations of GRB sources (e.g., Tavani 1998 and references therein).

The observations of new GRB afterglows continue to show new properties that contribute to making the interpretative scenario complex and incomplete. An interesting example is the so-called GRB-supernova connection. After the discovery by BeppoSAX of a GRB possibly associated with a type Ic supernova (GRB 980425, Pian et al. 2000; Galama et al. 1998), the possibility that some GRBs might be associated with supernovae has been proposed

Send offprint requests to: M. Feroci,

e-mail: feroci@ias.rm.cnr.it and supported by late-time observations of GRBs like 980326, 970228, 990712 and 970508 (Bloom et al. 1999; Reichart 1999; Hjorth et al. 1999; Sokolov 2001). The case for GRB 990712 is now debated (Sahu et al. 2000; Hjorth et al. 2000; Bjornsson et al. 2001).

A further interesting result recently provided by BeppoSAX is the identification of a potential new sub-class of GRBs: the Fast X-ray Transients (FXT, Heise et al. 2001). They are flashes of X-rays, so far not recurrent, sometimes accompanied by weak gamma-ray emission, that show no properties common to any known class of X-ray sources. The unique property of these events is their large $\mathrm{X}$-ray content, comparable to or dominant over their emission at gamma-rays. Their occurrence rate is approximately one third of the GRBs; they could be X-ray counterparts of a - so far unexplored - new class of very soft GRBs (Kippen et al. 2001; Heise et al. 2001). Attempts to find afterglows have failed so far. Fascinating proposals have been suggested to interpret them, going from "dirty fireball" GRBs (e.g., Dermer 1999) to highly redshifted classical GRBs.

In this paper we present the detection, localization and study of GRB 990704 and its X-ray afterglow. This event, the 17th GRB promptly localized by the BeppoSAX 
Wide Field Cameras and soon after observed with the BeppoSAX Narrow Field Instruments, is peculiar in many respects, and may be related to the FXT class.

\section{GRBM and WFC observations}

The gamma-ray burst GRB 990704 triggered the Gamma Ray Burst Monitor (GRBM, Frontera et al. 1997; Feroci et al. 1997; Costa et al. 1998) onboard BeppoSAX on 1999, July 4th 17:30:20.221 UT (63020.221 s of day, SOD) and was at the same time detected and imaged by unit 1 of the Wide Field Cameras (WFC, Jager et al. 1997). WFC data revealed the GRB with a signal-to-noise ratio of 18 at the position in the sky $(\mathrm{J} 2000) \mathrm{RA}=12^{\mathrm{h}} 19^{\mathrm{m}} 30^{\mathrm{s}}$ and $\mathrm{Dec}=-3^{\circ} 48$ '2, with an error radius (99\% confidence level) of $7^{\prime}$ (Heise et al. 1999). The relatively large size of the WFC error region is primarily due to an unfavorable satellite attitude. An independent position of the same event was obtained through the analysis of the difference in the arrival times of the event at the BeppoSAX GRBM and the Ulysses GRB detector. This resulted in a 2 '.8 wide $(3-\sigma)$ annulus in the sky intersecting the WFC error circle, which reduced the error box to $\sim 75 \operatorname{arcmin}^{2}$ (see Fig. 6) (Hurley \& Feroci 1999).

The light curve of the event as derived from the GRBM and WFC experiments is shown in different energy ranges in Fig. 1. The GRBM light curve is given in two separate energy ranges (40-100 keV and 100-700 keV). They are derived from the available GRBM data: 1-s count-rates in the two partially overlapping ranges $40-700 \mathrm{keV}$ and $>100 \mathrm{keV}$. Since the GRBM effective area above $700 \mathrm{keV}$ is very small, we can usually assume that the contribution to the count rate from photons with energy above $700 \mathrm{keV}$ is negligible, and we can obtain light curves in the two adjacent ranges $40-100 \mathrm{keV}$ and 100-700 keV (see Amati 1999 for an extensive discussion on this subject). This procedure has been verified in the specific case of GRB 990704 with the time-averaged spectral data available from the GRBM (e.g., Feroci et al. 1997). The 240channel energy spectra, in the full energy range from 40 to $700 \mathrm{keV}$, integrated over fixed 128-s time intervals, were used to derive the time-averaged spectral shape extrapolation beyond $700 \mathrm{keV}$, verifying that the number of counts expected above $700 \mathrm{keV}$ is negligible with respect to those detected in 40-100 keV. In the case of GRB 990704 we expect the counts above $700 \mathrm{keV}$ to be less than $0.3 \%$ of those detected in the $40-100 \mathrm{keV}$ range.

The peak fluxes and the fluences for GRB 990704 in different energy ranges are given in Table 1 . They can be used to compute the ratio of peak fluxes, namely $F_{\mathrm{X}} / F_{\gamma}=0.56 \pm 0.09$ using the $2-10 \mathrm{keV} \mathrm{X}$-ray flux and $1.56 \pm 0.23$ using the $2-26 \mathrm{keV} \mathrm{X}$-ray flux: the largest values found so far in the BeppoSAX sample (Fig. 2). The duration of the event is perhaps slightly energy dependent, ranging from $\sim 37 \mathrm{~s}$ in $2-5 \mathrm{keV}$ to less than $\sim 30 \mathrm{~s}$ above $100 \mathrm{keV}$. The ratios of fluences in X-rays and gamma-rays are again larger than any in the BeppoSAX sample (see Fig. 3): $S_{2-10 \mathrm{keV}} / S_{40-700 \mathrm{keV}}=1.52 \pm 0.15$

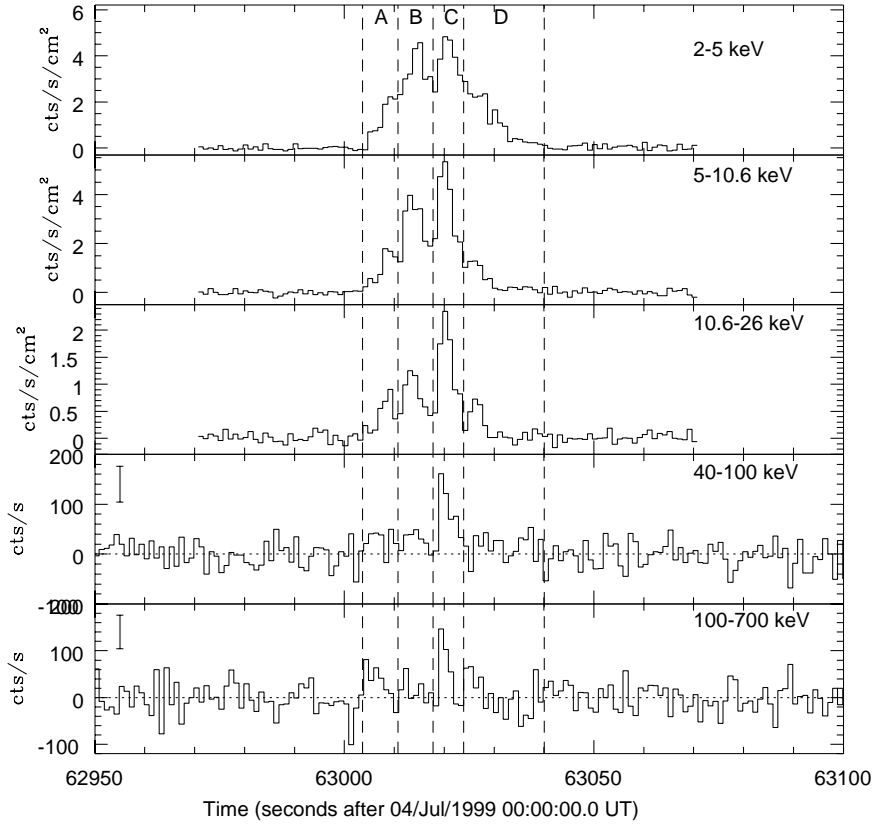

Fig. 1. WFC (top 3 panels) and GRBM (bottom 2 panels, on the top left the typical $\pm 1 \sigma$ uncertainty is given) light curves of GRB 990704. Vertical dashed lines identify the intervals for the time-resolved spectral analysis (see text).

Table 1. Peak fluxes (1-s) and fluences of GRB 990704 in different energy ranges.

\begin{tabular}{|l|l|l|l|}
\hline & $2-10 \mathrm{keV}$ & $2-26 \mathrm{keV}$ & $40-700 \mathrm{keV}$ \\
\hline $\begin{array}{l}\text { Peak Flux, } F \\
\left(10^{-7} \mathrm{erg} \mathrm{cm}^{-2} \mathrm{~s}^{-1}\right)\end{array}$ & $1.0 \pm 0.1$ & $2.80 \pm 0.28$ & $1.8 \pm 0.2$ \\
\hline $\begin{array}{l}\text { Fluence, } S \\
\left(10^{-6} \mathrm{erg} \mathrm{cm}^{-2}\right)\end{array}$ & $1.52 \pm 0.08$ & $2.84 \pm 0.14$ & $1.0 \pm 0.1$ \\
\hline
\end{tabular}

and $S_{2-26 \mathrm{keV}} / S_{40-700 \mathrm{keV}}=2.84 \pm 0.27$. As a comparison, if we consider the sample of the GRBs localized by BeppoSAX between July 1996 and December 1999, we obtain the average values and scatters provided in Table 2. The basic data for the computation were available only for subsets of the total number of events, specified in parentheses. A systematic analysis of the complete sample will be reported elsewhere (Frontera et al., in preparation). An examination of the quick-look data (that is, the raw data promptly analysed at the BeppoSAX Science Operation Center) for the events not included in our analysis, indicates that their exclusion should not significantly affect our results. Table 2 makes it very evident that GRB 990704 and 981226 are both responsible for a large increase of both the average and the scatter of the fluence ratios, whereas 990704 alone causes a significant increase in the average and scatter of the peak flux ratios.

The WFC did not detect X-ray emission from the location of GRB 990704 after the end of the GRB prompt emission. The $3-\sigma$ upper limit in the $2-10 \mathrm{keV}$ range in the time interval 63040-63076 s UT (that is, the $36 \mathrm{~s}$ after the end of the GRB) is $1.6 \times 10^{-9} \mathrm{erg} \mathrm{cm}^{-2} \mathrm{~s}^{-1}$. 
Table 2. The average, $\langle x\rangle$, and the standard deviation, $s$, of the $\mathrm{X}$ to gamma content of the sample of GRBs localized by BeppoSAX between 1996 and 1999. The total number of events is 28. For each of the estimates, the number of events for which the data were available is given in parentheses (see also text).

\begin{tabular}{|l|l|l|l|l|l|}
\hline & $\begin{array}{l}F_{2-10 \mathrm{keV}} / \\
F_{40-700 \mathrm{keV}}\end{array}$ & $\begin{array}{l}F_{2-26 \mathrm{keV}} / \\
F_{40-700 \mathrm{keV}}\end{array}$ & $\begin{array}{l}S_{2-10 \mathrm{keV}} / \\
S_{40-700 \mathrm{keV}}\end{array}$ & $\begin{array}{l}S_{2-26 \mathrm{keV}} / \\
S_{40-700 \mathrm{keV}}\end{array}$ & Notes \\
\hline$<x>$ & $0.15(9)$ & $0.29(16)$ & $0.31(17)$ & $0.63(16)$ & \\
\hline$s$ & $0.18(9)$ & $0.42(16)$ & $0.45(17)$ & $0.83(16)$ & \\
\hline$<x>$ & $0.10(8)$ & $0.20(15)$ & $0.23(16)$ & $0.47(15)$ & excluding 990704 \\
\hline$s$ & $0.10(8)$ & $0.26(15)$ & $0.33(16)$ & $0.60(15)$ & excluding 990704 \\
\hline$<x>$ & $0.08(7)$ & $0.18(14)$ & $0.15(15)$ & $0.34(14)$ & $\begin{array}{l}\text { excluding } 990704 \\
\text { and } 981226\end{array}$ \\
\hline$s$ & $0.08(7)$ & $0.26(14)$ & $0.13(15)$ & $0.28(14)$ & $\begin{array}{l}\text { excluding } 990704 \\
\text { and } 981226\end{array}$ \\
\hline
\end{tabular}
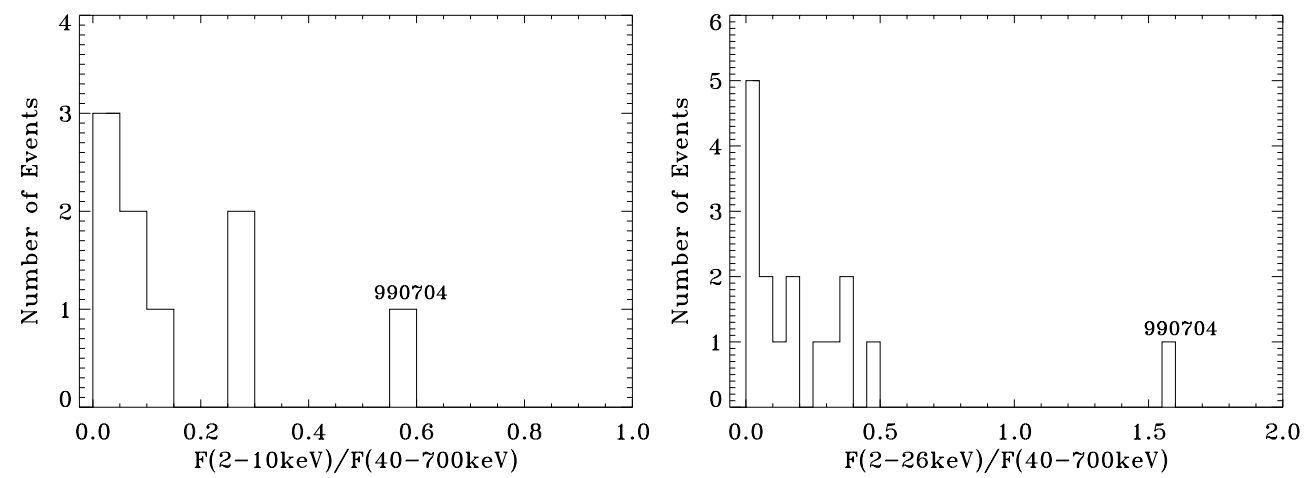

Fig. 2. Histogram of the X-to-gamma peak flux ratios, for the two X-ray energy ranges $2-10$ and 2-26 keV, of the same events used to derive Table 2 .
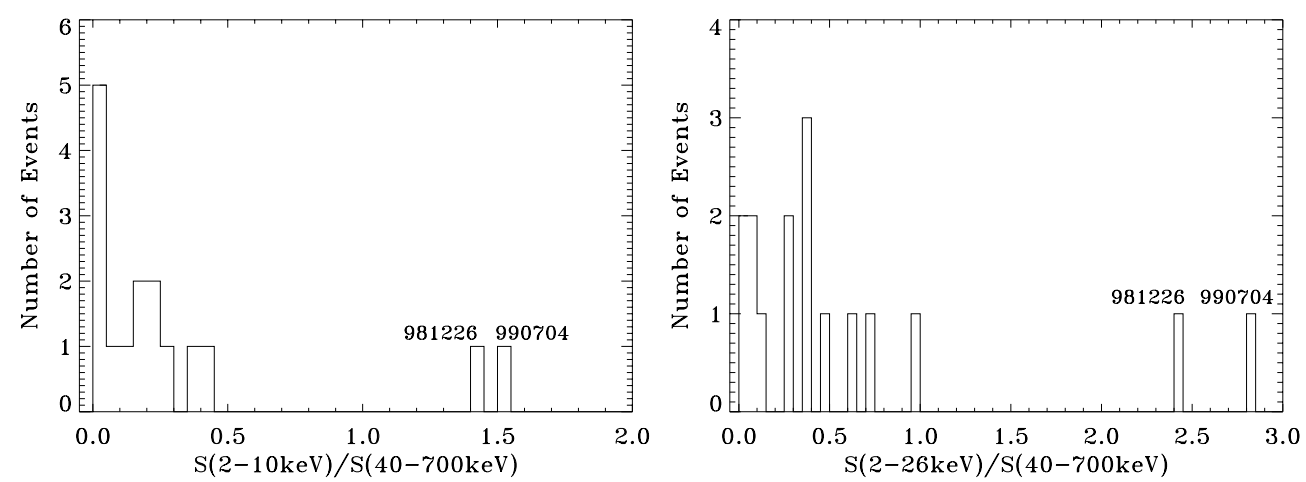

Fig. 3. X-to-gamma fluence ratios, for the two X-ray energy ranges $2-10$ and $2-26 \mathrm{keV}$, of the same events used to derive Table 2.

Only one of the four X-ray peaks has a significant gamma-ray counterpart, the one included in interval $\mathrm{C}$ in Fig. 1; it is also the most intense in the $\mathrm{X}$-ray range. The shape of the gamma-ray pulse exhibits a profile consistent with a FRED (Fast Rise Exponential Decay) profile. We checked the energy dependence of the width of this pulse. For this purpose, we used the high time resolution data available from both the WFC and the GRBM, binned at $0.5 \mathrm{~s}$ (Fig. 4). We could derive a value of the duration (in terms of full width at half maximum, $F W H M$ ) of the main peak only in four energy ranges because the high time resolution data from the GRBM are only available in the integrated energy range $40-700 \mathrm{keV}$, and they start $8 \mathrm{~s}$ before the trigger time. The estimated $F W H M$ values are shown in Fig. 5. We found that the width uncertainties were comparable to the time resolution. Although the multi-peaked structure observed at X-rays might significantly affect our estimates, the derived values appear in agreement with the $E^{-0.45}$ ( $E$ photon energy) dependence found by Fenimore et al. (1995) for the classical GRBs (e.g., Piro et al. 1998 and references therein). 


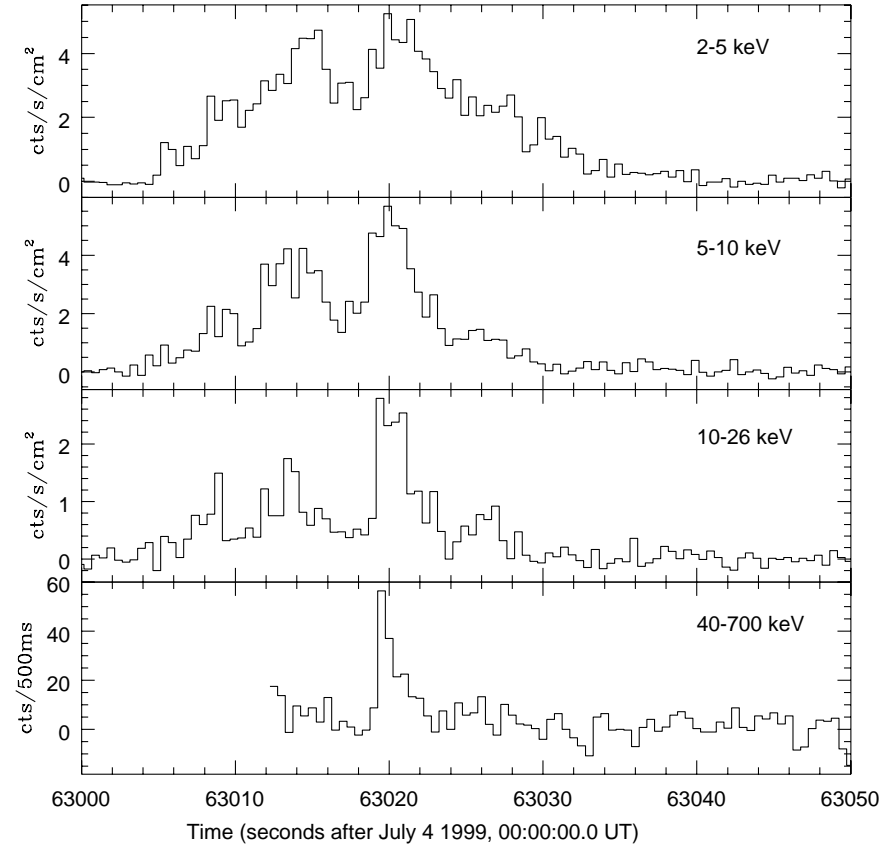

Fig. 4. Light curve of GRB 990704 with $500 \mathrm{~ms}$ time resolution, providing a clear view of the energy dependence of the width of the pulse at $63020 \mathrm{~s}$ UT. For the GRBM data, only the integrated $40-700 \mathrm{keV}$ energy range is available with this time resolution.

\subsection{Spectral analysis}

We analyzed the energy spectrum of the GRB event using the WFC and GRBM data. To perform a time-resolved spectral analysis, for the GRBM we used the two-channel spectra that can be derived from the two energy ranges of the 1-s ratemeters (Amati 1999). The two available channels cover the energies 40-100 and 100-700 keV. We selected four time intervals (A, B, C, D; Fig. 1) during the event, encompassing the four distinct peaks visible in the energy range $11-26 \mathrm{keV}$ in the WFC data. The GRBM high energy (100-700 keV) channel does not provide a significant detection in any of the four selected intervals, and this datum was therefore excluded during the spectral fitting procedure.

We first attempted to model the spectrum in the four intervals with a simple (unabsorbed) power law. This solution is acceptable only for the interval D (see Table 3), for which intrinsic photoelectric absorption is not needed. For the other intervals different models were needed. In particular, the average spectrum of interval A can be satisfactorily fit either by adding photoelectric absorption by cold matter or changing the spectral model to a broken power law. In intervals $\mathrm{B}$ and $\mathrm{C}$ an absorbed power law is not acceptable, whereas the broken power law is satisfactory.

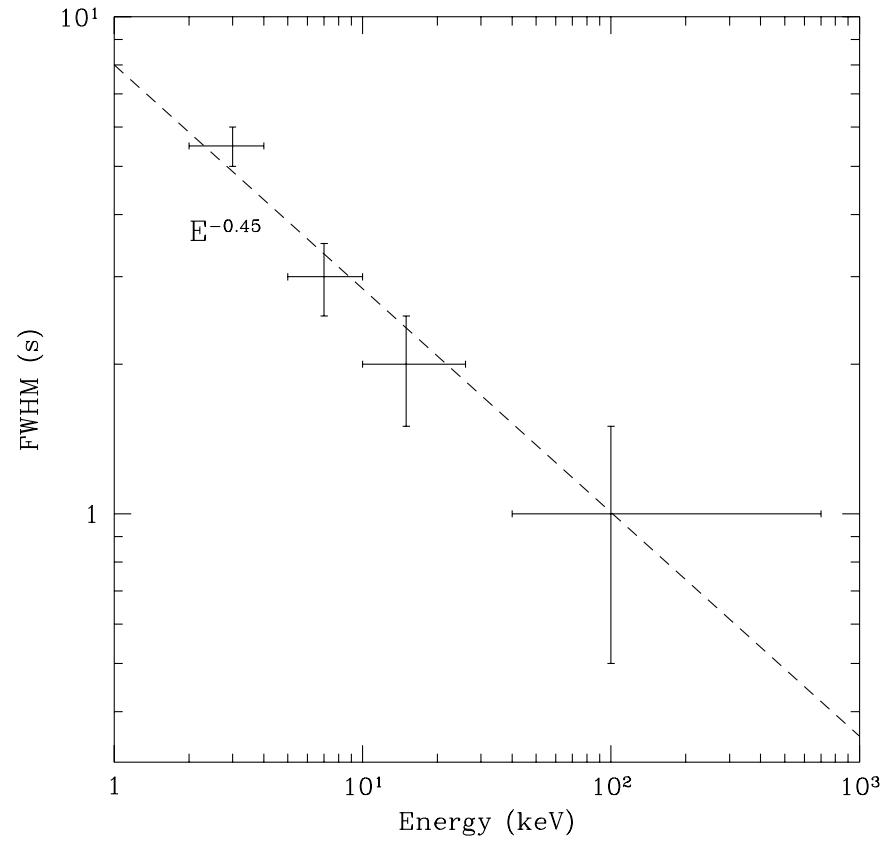

Fig. 5. Full Width at Half Maximum of the main peak of GRB 990704 (see temporal slice C in Fig. 1), as a function of energy. The dashed line shows a $F W H M \propto E^{-0.45}$ relation. Horizontal error bars indicate the energy band. Vertical error bars represent $500 \mathrm{~ms}$ (see text and Fig. 4), they must be taken only as an indication of the uncertainty on the duration estimate.

Table 3. Time-resolved spectral analysis of GRB 990704. The spectral model is either a simple power law (photon index $\alpha$ ), an absorbed power law, or a broken power law (low and high energy photon indices $\alpha$ and $\beta$, break energy $\left.E_{\text {break }}\right)$. Errors are given at $90 \%$ confidence level. Asterisks $\left(^{*}\right)$ indicate parameters for which an error could not be derived because of a large $\chi^{2}$.

\begin{tabular}{|l|l|l|l|l|l|}
\hline & $\alpha$ & $E_{\text {break }}(\mathrm{keV})$ & $\beta$ & $N_{\mathrm{H}} \times 10^{22} \mathrm{~cm}^{-2}$ & $\chi_{\nu}^{2}$ (d.o.f.) \\
\hline $\mathrm{A}$ & $2.12_{-0.14}^{+0.16}$ & - & - & $4.85_{-3.14}^{+4.25}$ & $0.93(13)$ \\
& $1.31_{-0.98}^{+0.34}$ & $11.4_{-7.1}^{+50.2}$ & $2.35_{-0.32}^{+4.14}$ & - & $0.80(10)$ \\
\hline $\mathrm{B}$ & $2.55^{*}$ & - & - & $8.7^{*}$ & $2.16(12)$ \\
& $0.92_{-0.34}^{+0.30}$ & $7.4_{-1.1}^{+2.3}$ & $2.70_{-0.16}^{+0.21}$ & - & $1.04(11)$ \\
\hline $\mathrm{C}$ & $2.14_{-0.07}^{+0.08}$ & - & - & $4.23_{-1.61}^{+1.86}$ & $1.43(12)$ \\
& $1.29_{-0.27}^{+0.22}$ & $9.3_{-2.3}^{+41.9}$ & $2.29_{-0.13}^{+2.75}$ & - & $0.87(11)$ \\
\hline $\mathrm{D}$ & $2.14_{-0.11}^{+0.14}$ & - & - & $<2.55$ & $1.15(12)$ \\
& $2.11_{-0.09}^{+0.10}$ & - & - & - & $1.09(13)$ \\
\hline
\end{tabular}

\section{The X-ray afterglow}

\subsection{Observations}

Soon after the localization of GRB 990704 by the WFC a Target of Opportunity observation with the BeppoSAX Narrow Field Instruments (NFI, Boella et al. 1997) was initiated. The NFI observation began on July 5, 01:48 UT, approximately eight hours after the GRB, and was initially scheduled to last for $100 \mathrm{ks}$ of net observing time. However, following the GRBM/WFC detection and localization of GRB 990705 (e.g., Amati et al. 2000) a new 


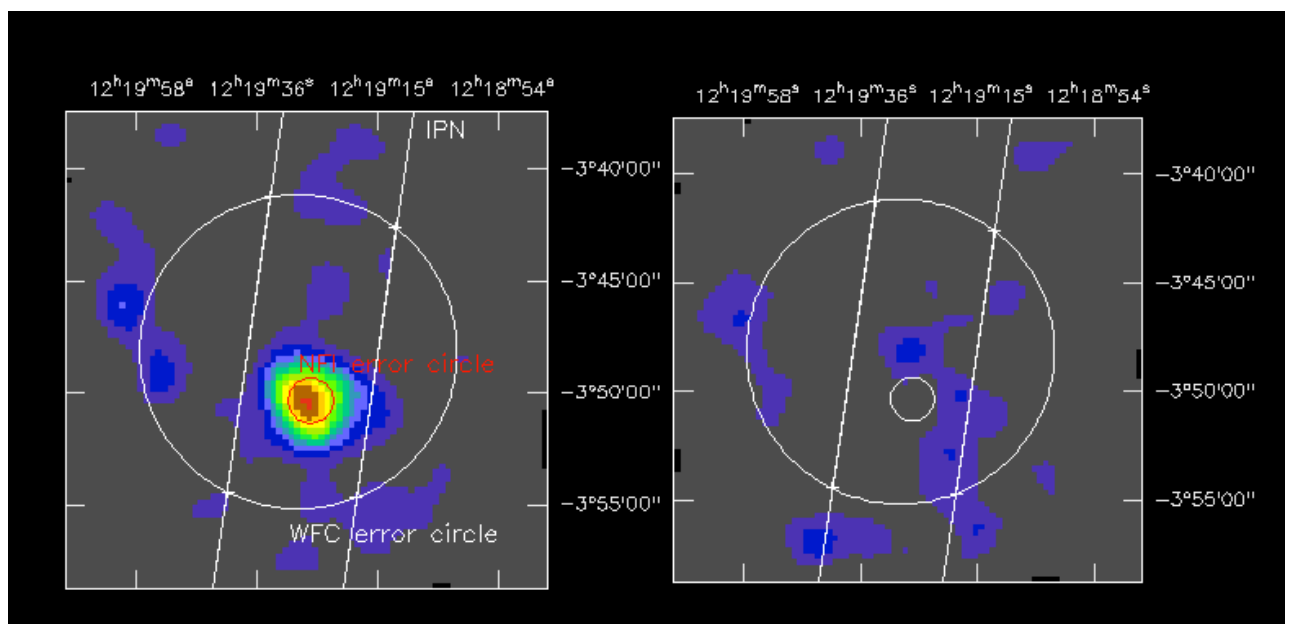

Fig. 6. MECS image of the field of GRB 9907048 hours after the burst (left) and one week later (right). The fading X-ray source is 1SAX J1219.5-0350. The WFC, IPN and NFI error boxes are indicated.

Target of Opportunity was declared on the latter GRB and the NFI observation of the field of GRB 990704 was stopped on July 5, 23:45 UT for a total net exposure time of $37 \mathrm{ks}$ for the MECS and $13.4 \mathrm{ks}$ for the LECS.

A previously unknown X-ray source, 1SAX J1219.50350, was detected almost at the center of the MECS and LECS detectors, at the sky position (J2000) RA = $12^{\mathrm{h}} 19^{\mathrm{m}} 27^{\mathrm{s}} .3$ and $\mathrm{Dec}=-3^{\circ} 50^{\prime} 22^{\prime \prime}$ (90\% confidence level error radius 1'), consistent with the GRB 990704 combined error box (Fig. 6, Feroci et al. 1999; Hurley \& Feroci 1999). The source flux, averaged over the entire NFI observation, was (5.64 \pm $0.45) \times 10^{-3}$ counts $\mathrm{s}^{-1}$ in the MECS $(1.6-10 \mathrm{keV})$ and $(3.2 \pm 0.8) \times 10^{-3}$ counts $\mathrm{s}^{-1}$ in the LECS $(0.1-$ $2 \mathrm{keV}$ ) (uncertainties on the fluxes are 1- $\sigma$ ).

Given that the X-ray flux from 1SAX J1219.5-0350 appeared to decrease with time during the observation, a new NFI pointing was scheduled at its position in order to verify its association with GRB 990704. This second NFI observation started on July 11, 19:10 UT and ended on July 12, 20:48 UT, for a net MECS exposure time of $42.5 \mathrm{ks}$. We averaged the flux over both the entire second pointing and also over its first $17 \mathrm{ks}$, and found no detection of the source either in the MECS or in the LECS. We derived a $3-\sigma$ upper limit of $9.4 \times 10^{-4}$ counts s $^{-1}$ (MECS, 2-10 keV, $42.5 \mathrm{ks}$ ).

Based on the angular consistency with the GRB location and the temporal behaviour, similar to that of previous GRB afterglows, we identify 1SAX J1219.5-0350 as the X-ray afterglow of GRB 990704.

Although the event occurred at high Galactic latitude and the WFC and NFI error boxes were promptly searched, no optical or radio afterglow was detected (Castro-Tirado et al. 1999; Maury et al. 1999; Diercks et al. 1999; Vrba et al. 1999). In the optical $R$ band the tightest upper limit to a variable object is at $R=22.5$ within less than 5 hours after the burst (Jensen et al. 1999) and $R \sim 23.5$ (Rol et al. 1999) after 30.5 hours.
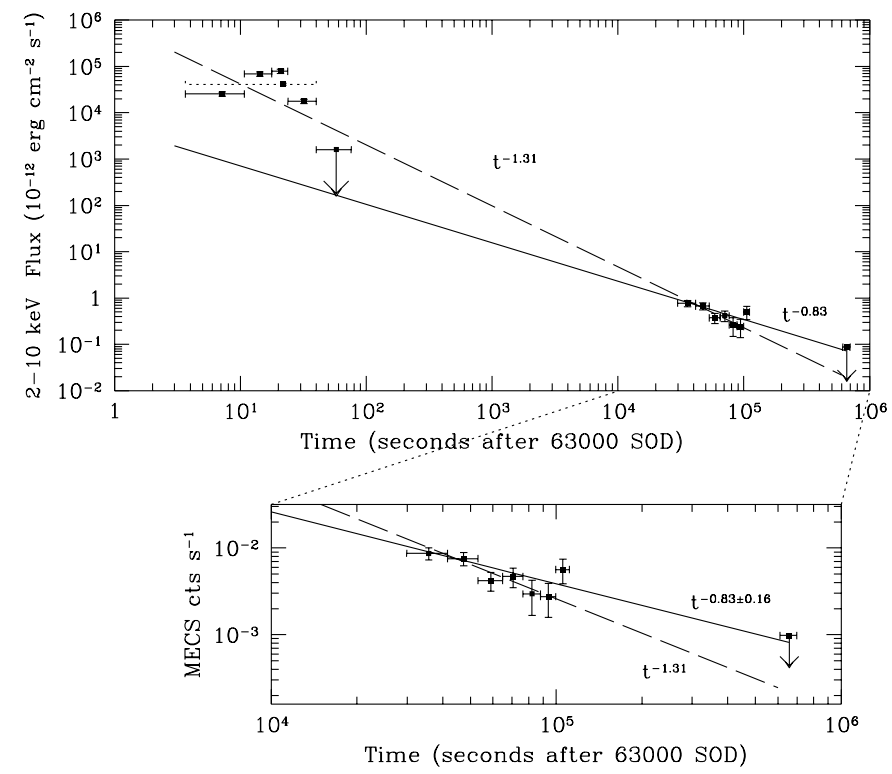

Fig. 7. Decay of the X-ray afterglow of GRB 990704, as energy flux (upper panel) and photons flux (lower panel).

\subsection{Data analysis}

The MECS 2-10 keV light curve of the first observation was accumulated with time bins of $11640 \mathrm{~s}$, using an extraction radius of $2^{\prime}$ around the source centroid. The background in the same bandpass was extracted at two different positions in the same field, with extraction radii of $2^{\prime}$ as well. The background is in both cases consistent with a constant value of $1.8 \times 10^{-3}$ counts $\mathrm{s}^{-1}$ and an analysis over the field shows no systematic trend. To derive the MECS background-subtracted light curve in Fig. 7, we used the average of the two background regions at each time bin. A constant value for the background yelded consistent results. In Fig. 7 (bottom panel) we show the temporal behaviour of the X-ray flux $(2-10 \mathrm{keV})$ from 1 SAX J1219.5-0350 in units of MECS counts $\mathrm{s}^{-1}$. 
The photon spectrum from the LECS and MECS instruments has been fit with a simple power law, $I(E)=$ $C E^{-\Gamma}$ photons $\mathrm{s}^{-1} \mathrm{~cm}^{-2} \mathrm{keV}^{-1}$. The LECS data were used in the energy range between 0.16 and $4.0 \mathrm{keV}$, where the response matrix is most accurate, while the MECS data were used in $1.6-10.0 \mathrm{keV}$. The result of the fit is $\Gamma=$ $\left(1.69_{-0.34}^{+0.60}\right)$ (reduced $\chi^{2}=1.83,17$ d.o.f.), giving an average flux in $2-10 \mathrm{keV}$ of $(5.0 \pm 0.4) \times 10^{-13} \mathrm{erg} \mathrm{cm}^{-2} \mathrm{~s}^{-1}$ and $(2.96 \pm 0.57) \times 10^{-13} \mathrm{erg} \mathrm{cm}^{-2} \mathrm{~s}^{-1}$ in $0.16-2.0 \mathrm{keV}$. The absorption at low energies is not very well constrained but appears consistent with that expected from absorption by cold interstellar gas along the line of sight as interpolated from the HI-maps by Dickey \& Lockman (1990) which results in $N_{\mathrm{H}}=3 \times 10^{20} \mathrm{~cm}^{-2}$. Our spectral results are in agreement with those obtained by Yonetoku et al. (2000) using ASCA data on the same afterglow.

We also performed the spectral analysis of the LECS and MECS data dividing the first observation in two portions of similar duration, in order to check for spectral evolution. We used an absorbed simple power law model to fit the data and we got satisfactory results in both parts. In the first spectrum the best fit value for the power law photon index is $\Gamma=(1.78 \pm 0.45)$ (reduced $\chi^{2}=1.02$, 8 degrees of freedom). For the second part of the observation we obtained $\Gamma=\left(1.48_{-0.67}^{+0.75}\right)$ (reduced $\chi^{2}=1.04$, 5 d.o.f.). Uncertainties on $\Gamma$ are given at $90 \%$ confidence for one parameter. In both cases the hydrogen absorption column is consistent with the average Galactic value.

The average MECS spectrum was used to convert the afterglow light curve from counts $\mathrm{s}^{-1}$ to $\mathrm{erg} \mathrm{cm}^{-2} \mathrm{~s}^{-1}$. The resulting light curve is shown in the top panel of Fig. 7, together with the $2-10 \mathrm{keV}$ WFC flux during the GRB prompt event averaged over the whole observation (dotted horizontal bar) and time-resolved in the four intervals as indicated in Fig. 1, and the upper limit obtained from WFC data during the $\sim 36 \mathrm{~s}$ after the event. The light curve during the first NFI observation is well described by a power-law decay $I(t) \propto t^{-\beta}$, with $\beta=0.83 \pm 0.16$. However, including in the fit the average X-ray flux measured by the WFC during the GRB yields $\beta=1.31$. Both indices are consistent with the NFI upper limit during the second observation, but only the flatter one is consistent with the WFC upper limit soon after the event. On the other hand, the back extrapolation of the law $I(t) \propto t^{-0.83}$ largely underestimates the prompt X-ray emission.

An ASCA observation of the same X-ray afterglow continued $12 \mathrm{ks}$ beyond the BeppoSAX one (Murakami et al. 1999). An early suggestion of a flare in the X-ray flux at the end of the ASCA observation (Yoshida et al. 1999a) was not confirmed by a later analysis (Murakami et al. 2000; Yonetoku et al. 2000). We searched the last portion of our data for the beginning of such a flare and found a marginal indication of flux rise during the last two orbits of our observation. Flaring of the X-ray afterglow has been observed in two events in the past: GRB 970508 (Piro et al. 1999) and GRB 970828 (Yoshida et al. 1999b). In both cases there were hints of a redshifted Fe-K emission line in the X-ray spectrum, at the time of the flare. We searched for spectral features in our spectrum, both for the whole observation and for its last part. No evidence is found for an emission line. Yonetoku et al. (2000) have used the ASCA data on GRB 990704 to set a tight upper limit (equivalent width smaller than $\sim 250 \mathrm{eV}$ ) to possible Fe-K emission line.

\section{Discussion}

Several characteristics of GRB 990704 appear noteworthy. Regarding the GRB prompt emission:

1) The first portion of the event appears dominated by the X-ray emission (see Fig. 1). Only the main peak is distinctively detected at energies above $100 \mathrm{keV}$.

2) The X-ray content of the prompt emission is extremely high, compared to the other BeppoSAX GRBs. Figures 2 and 3 show how distinct GRB 990704 is with respect to the distribution of the other events within the BeppoSAX GRB sample mentioned in Sect. 2. In fact, the second largest value found in our sample for $P_{2-26 \mathrm{keV}} / P_{40-700 \mathrm{keV}}$ is 0.45 for GRB 981226 (whereas 990704 shows a value of 1.56). In terms of fluences, we find $S_{2-26 \mathrm{keV}} / S_{40-700 \mathrm{keV}}=2.84$ for 990704 , 2.40 for 981226 and 0.95 for the largest, 980326 , in the remaining sample. That is, for GRB 990704 the energy recorded in 2-26 $\mathrm{keV}$ is almost 3 times that recorded in 40-700 keV. From this point of view, GRB 990704 hardly meets the classical GRB defining criteria. Still, it showed an X-ray afterglow as do "classical" GRBs.

3) The main peak observed in gamma-rays (i.e., the third one in X-rays) appears to be consistent with the typical energy dependence of the pulse width $(\Delta t \propto$ $E^{-0.45}$ ) observed in many individual GRB pulses (Fenimore et al. 1995). This dependence is consistent with a scenario in which the prompt emission is due to synchrotron radiation in a shocked medium (e.g., Tavani 1996). From this point of view GRB 990704 appears to be a "classical" GRB.

The X-ray afterglow of GRB 990704 is peculiar. The BeppoSAX measurement shows a very slow decay, with index $\sim 0.8$. If we consider a sample of thirteen BeppoSAX X-ray afterglows (Stratta et al., in preparation) from 1997 to May 1999, we find that the average value of the power law decay slopes is 1.36 and the standard deviation is only 0.16 . However, a word of caution: a power law decay with a slope of only 0.8 , if extrapolated to an infinite time would cause the total emitted energy to diverge. Therefore, we have to assume that after the end of the BeppoSAX observation the decay slope changed to a larger value. In this respect, recall the claim (but not confirmed by a later analysis) of an ASCA detection of rebursting of the $\mathrm{X}$-ray flux from this source in the few hours following the end of the BeppoSAX observation. If such a rebursting occurred, then the slow decay measured by BeppoSAX could be an artifact of an incomplete sampling of the bursting behaviour of the X-ray flux. In this case the true overall 
decay would be indeed faster, possibly the $\sim 1.3$ slope we obtain taking into account the prompt X-ray emission.

The softness of the prompt emission from GRB 990704 is definitely peculiar. As shown in Fig. 3, a similarly large $\mathrm{X}$-to-gamma energy ratio was only observed once in the BeppoSAX GRB sample, in the case of GRB 981226. It too was peculiar in its $\mathrm{X}$-ray afterglow, showing an initial short rise, or maybe a plateau, never seen in any other X-ray afterglow (Frontera et al. 2000a). In the GINGA sample of 22 GRBs (Strohmayer et al. 1998), only one event, GRB 900901, had a X-to- $\gamma$ fluence ratio comparable to GRB 990704. In that case the ratio was 1.23 , although the gamma-ray fluence was measured in $50-300 \mathrm{keV}$, and this most likely slightly biases upward the value of the ratio when compared to our sample.

In a few other BeppoSAX GRBs the ratio of $\mathrm{X}$ - to gamma-ray fluence was relatively high (up to 0.4 ) with respect to the less than $\sim 10 \%$ usually observed in GRBs. For example, see Table 3 in Frontera et al. (2000b) and Fig. 3 for the BeppoSAX events, and Table 1 in Strohmayer et al. (1998) for GINGA, where the average value of $S_{2-10 \mathrm{keV}} / S_{50-300 \mathrm{keV}}$ goes down to $\sim 12 \%$ if one excludes just the largest 3 values. It is intriguing to note that the events in the BeppoSAX sample with a high X-ray content in their prompt emission include all five GRBs proposed to be associated with supernovae, on the basis of the properties of their optical afterglow. In fact, they showed the following values for $S_{2-10 \mathrm{keV}} / S_{40-700 \mathrm{keV}}$ (Frontera et al. 2000b; Frontera et al. 2001): 980425: 28\%; 980326: 38\%; 970228: 20\%; 990712: 40\%; and 970508: 20\%. Taken individually, these are not extraordinary values but it is suggestive that all of them have values larger than the average. Actually, as Frontera et al. (2000a) suggested, the explosion of a supernova prior to the GRB may enrich the circumburst medium with baryon-rich matter. This would provide a natural explanation for a lower Lorentz factor of the expanding fireball following the GRB explosion, leading to the observed lower peak energy of the emitted radiation and consequently the X-ray richness in this type of GRBs. We note, however, that in some of the cases mentioned above (e.g., 980425 and 980326) the assumption is that the supernova exploded right at the time of the GRB, so that this scenario may not apply in a straightforward way.

Finally, we note that GRB 990704 is definitely the BeppoSAX GRB with characteristics that are the closest to the recently suggested class of FXTs. As mentioned in the introductory section, these events are not yet firmly characterized. Based on the BeppoSAX data (Heise et al. 2001), so far we know that they are almost indistinguishable from X-ray counterparts of classical GRBs (in terms of duration, hardness, flux), but they do not show gamma-ray emission detectable by the GRBM. Quantitatively, the X-to-gamma peak flux ratios for FXTs go from few to a hundred, while the ratio of fluences goes from 0.1 to 10 (Heise et al. 2001). Therefore, GRB 990704 appears as the classical GRB (because it has a GRBM-detected emission and an
X-ray afterglow) within the BeppoSAX sample that most closely approaches the properties of the FXTs. Should the $\mathrm{X}$-ray content be the signature of a supernova, then supernova explosions would be good candidates for the origin of FXTs.

More observations will be needed to give (or not) observational support to our conjectures but the current data are intriguing, and theoreticians are already at work on similar ideas, e.g., Woosley (2001).

Acknowledgements. The authors would like to remember the key role of Daniele Dal Fiume in the BeppoSAX project, and the GRB project in particular. They are grateful to Chris Butler, former BeppoSAX Mission Director, and to the Teams at the BeppoSAX Science Operation Center, Operation Control Center and Science Data Center for their enthusiastic contribution to the BeppoSAX GRB program. The authors warmly thank Hale Bradt for suggestions and for a careful reading of the manuscript. They also thank the anonymous referee for comments that improved the paper. BeppoSAX is a program of the Italian Space Agency (ASI) with participation of the Netherlands Agency for Aerospace Programs (NIVR).

\section{References}

Amati, L. 1999, Ph.D. Thesis, available at http://tonno.tesre.bo.cnr.it/ amati/tesi/

Amati, L., Frontera, F., Vietri, M., et al. 2000, Science, 290, 953

Bjornsson, G., Hjorth, J., Jakobsson, P., Christensen, L., \& Holland, S. 2001, ApJ, 552, L121

Boella, G., Butler, R. C., Perola, G. C., et al. 1997, A\&AS, 122,299

Bloom, J. S., Kulkarni, S. R., Djorgovski, S. G., et al. 1999, Nature, 401, 453

Castro-Tirado, A. J., Alcoholado-Feltstrom, M. E., Marcha, M., et al. 1999, GCN Circ., No. 362

Costa, E., Frontera, F., Heise, J., et al. 1997, Nature, 387, 783

Costa, E., Frontera, F., Dal Fiume, D., et al. 1998, Adv. Sp. Res., 22, 1129

Costa, E. 2000, AIP, 526, 365

Dermer, C. D. 1999, A\&AS, 138, 519

Dickey, \& Lockman 1990, ARA\&A, 28, 215

Diercks, A., Krisciunas, K., \& Deutsch, E. 1999, GCN Circ., No. 370

Fenimore, E. E., in 't Zand, J. J. M., Norris, J. P., Bonnell, J. T., \& Nemiroff, R. J. 1995, ApJ, 448, L101

Feroci, M., Frontera, F., Costa, E., et al. 1997, SPIE Proc., 3114, 186

Feroci, M., Gandolfi, G., Antonelli, A., et al. 1999, IAU Circ., No. 7217

Frail, D., Kulkarni, S. R., Nicastro, L., Feroci, M., \& Taylor, G. B. 1997, Nature, 389, 261

Frontera, F., Costa, E., Dal Fiume, D., et al. 1997, A\&AS, 122, 357

Frontera, F., Antonelli, A., Amati, L., et al. 2000a, ApJ, 540, 697

Frontera, F., Amati, L., Costa, E., et al. 2000b, ApJS, 127, 59

Frontera, F., Amati, L., Vietri, M., et al. 2001, ApJ, 550, L47

Galama, T. J., Vreeswijk, P. M., van Paradijs, J., et al. 1998, Nature, 395, 670

Heise, J., in 't Zand, J., Celidonio, G., et al. 1999, IAU Circ., No. 7217 
Heise, J., in 't Zand, J., Kippen, R. M., \& Woods, P. 2001, Proc. 2nd Workshop Gamma Ray Bursts in the Afterglow Era, ed. E. Costa, F. Frontera, \& J. Hjorth

Hjorth, J., Fynbo, J., Dar, A., Courbin, F., \& Moller, P. 1999, GCN Circ., 403

Hjorth, J., Holland, S., Courbin, F., et al. 2000, ApJ, 534, L147

Hurley, K., \& Feroci, M. 1999, GCN Circ., No. 379

Jager, R., Mels, W. A., Brinkman, A. C., et al. 1997, A\&AS, 125,557

Jensen, B. L., Hjorth, J., Pedersen, H., et al. 1999, GCN Circ., No. 371

Kippen, M., Woods, P, Heise, J., et al. 2001, Proc. 2nd Workshop Gamma Ray Bursts in the Afterglow Era, ed. E. Costa, F. Frontera, \& J. Hjorth

Lamb, D. Q., preprint [astro-ph/0005028]

Maury, A., Gaillard, B., \& Boer, M. 1999, GCN Circ., Nos. 363 and 369

Murakami, T., Yoshida, A., Kawai, N., et al. 1999, GCN Circ., No. 372

Murakami, T., et al. 2000, talk given at the 2nd Workshop Gamma Ray Bursts in the Afterglow Era

Pian, E., Amati, L., Antonelli, A., et al. 2000, ApJ, 536, 778

Piro, L., Amati, L., Antonelli, A., et al. 1998, A\&A, 331, L41
Piro, L., Costa, E., Feroci, M., et al. 1999, ApJ, 514, L73

Reichart, D. 1999, ApJ, 521, L111

Rol, E., Vreeswijk, P., Galama, T., et al. 1999, GCN Circ., No. 374

Sahu, K. C., Vreeswijk, P., Bakos, G., et al. 2000, ApJ, 540, 74

Sokolov, V. 2001, Proc. 2nd Workshop Gamma Ray Bursts in the Afterglow Era, ed. E. Costa, F. Frontera, \& J. Hjorth

Strohmayer, T. E., Fenimore, E. E., Murakami, T., \& Yoshida, A. 1998, ApJ, 500, 873

Tavani, M. 1996, ApJ, 466, 768

Tavani, M. 1998, ApJ, 497, L21

van Paradijs, J., Groot, P. J., Galama, T., et al. 1997, Nature, 386,686

Vrba, F. J., Henden, A. A., Luginbuhl, C. B., et al. 1999, GCN Circ., Nos. 365 and 367

Woosley, S. 2001, Proc. 2nd Workshop Gamma Ray Bursts in the Afterglow Era, ed. E. Costa, F. Frontera, \& J. Hjorth

Yonetoku, D., Murakami, T., Ueda, Y., et al. 2000, PASJ, 52, 509

Yoshida, A., et al. 1999a, talk given at the 5th Huntsville GRB Symp.

Yoshida, A., Mihara, T., Matsuoka, M., et al. 1999b, A\&AS, 138, 433 